\title{
Health Promotion in Elderly People
}

The WHO has defined ideal health for elderly individuals as a state of functional independence. In terms of functional abilities, $5 \%$ of elderly can be classified as disabled, $\mathbf{2 0 \%}$ as fragile and $\mathbf{7 5 \%}$ as independent. Japanese society is aging at an extremely rapid pace, however, it lags behind in the implementation of measures for health promotion, particularly for independent elderly. From the standpoint of the maintenance of a vital society, it is a pressing social need to establish appropriate measures for such individuals. Among local governments, increasing medical and healthcare costs are thus causing growing financial problems. In addition to this, there is fear that serious social problems, such as a decrease in the labor force, will result from a declining birthrate. Under such circumstances, the QOL has therefore been increasingly focused on in the study of the health of the elderly. In the future, however, it is necessary to establish a concept of elderly "productivity" (regarding employment, free labor, volunteer activity, etc.), which extends beyond the traditional idea that independent elderly individuals can contribute to society. Based on this concept, practical measures should be taken by both individuals (i.e. citizens) and the community (i.e. society, government) to foster health promotion, an idea advocated by the WHO, which concerns the maintenance and improvement of the physical and mental health of the elderly.

Reflecting on such a situation, this special issue presents the results of A Study of regional systems for the elderly which are useful for the maintenance and improvement of the functional abilities and the promotion of social participation, a research project undertaken during the 6-year period between 2000 and 2005 with the support of a grant for the promotion of science and technology from the Ministry of Education, Culture, Sports, Science and Technology. The goal of this research is to develop regional methods and societal skills for the promotion of health in an aging society in the 21 st century by 1 ) attempting to establish a health index for the extension of a healthy life span, encourage social participation and the development of programs for the promotion and maintenance of health; 2) developing an effective, safe, and practical health promotion support system for residents in the region and evaluating the effects of such programs in a variety of fields; and 3) identify effective methods for establishing and publicizing such a system with the goal of increasing the knowledge of its existence in various communities which contain diversified backgrounds. It is hoped that these research results will therefore positively contribute to the improvement of society.

[International Journal of Sport and Health Science Vol.4, 303, 2006]

\begin{tabular}{|c|c|}
\hline \multicolumn{2}{|c|}{ PROFILE } \\
\hline \multicolumn{2}{|l|}{ 1. Name: } \\
\hline \multicolumn{2}{|l|}{ Hiroaki Tanaka, Ph.D. } \\
\hline \multicolumn{2}{|l|}{ 2. Address: } \\
\hline \multicolumn{2}{|l|}{ Department of Sports and Health Sciences } \\
\hline \multicolumn{2}{|l|}{ Fukuoka University } \\
\hline \multicolumn{2}{|l|}{ 8-19-1, Nanakuma, Jyonanku, Fukuoka, Japan } \\
\hline \multicolumn{2}{|l|}{$\mathrm{Tel} ;+81-92-871-6631$, ext 6767} \\
\hline \multicolumn{2}{|l|}{ Fax; +81-92-862-3033 } \\
\hline \multirow{2}{*}{\multicolumn{2}{|c|}{$\begin{array}{l}\text { e-mail; htanaka@fukuoka-u.ac.jp } \\
\text { 3. Vita: }\end{array}$}} \\
\hline & \\
\hline 1987 Ph.D. in Medicine. Ehime University, Ehime & 1. Yoshioka M, Ayabe M, Yahiro T, Higaki Y, St-Amand J, Miyazaki \\
\hline 1988- Professor, Laboratory of Fukuoka University, Fukuoka & H, Yoshitake Y, Shindo M, Tanaka H: Long-period accelerometer \\
\hline 2003-Director, Graduate school of Sports and Health & monitoring shows the role of physical activity in overweight and \\
\hline Science, Fukuoka University & obesity. Int. J.Obes Relat. Metab. Disord. 29:502-508, 2005 \\
\hline 4. Research field: & 2. Nishida Y, Tokuyama K, Nagasaka S, Higaki Y, Kiyonaga A, \\
\hline Exercise Physiology & Shindo M, Kusaka I, Nakamura T, Ishibashi S, Tanaka H. : Effect \\
\hline 5. Memberships of Academic Societies: & of moderate exercise training on peripheral glucose effectiveness, \\
\hline Japanese Society of Physical Fitness and Sports Medicine; Director & insulin sensitivity, and endogenous glucose production in healthy \\
\hline Japan Society of Health Promotion; Director & humans estimated by a two-compartment-labeled minimal model. \\
\hline Member of American College of Sports Medicine & Dlabetes 53:315-320, 2004 \\
\hline
\end{tabular}

